\title{
GPDs, the structure of the proton and wide-angle Compton scattering
}

\author{
P. Kroll \\ Fachbereich Physik, Universität Wuppertal, D-42097 Wuppertal, Germany
}

\begin{abstract}
Results from a recent analysis of the zero-skewness generalized parton distributions (GPDs) for valence quarks are reviewed. The analysis bases on a physically motivated parameterization of the GPDs with a few free parameters adjusted to the nucleon form factor data. Results for the GPDs are presented and a number of applications such as moments, Ji's sum rule or their impact parameter representation are discussed. The $1 / x$ moments, in particular, form the soft physics input to Compton scattering off protons within the handbag approach. The Compton cross section evaluated from this information is found to be in good agreement with experiment.

Talk presented at the workshop on the Shape of Hadrons, Athens (2006)
\end{abstract}

Keywords: generalized parton distributions, electromagnetic form factors, Compton scattering PACS: $12.38 \mathrm{Bx}, 12.39 \mathrm{St}, 13.40 \mathrm{Ks}, 13.60 \mathrm{Fz}$

\section{INTRODUCTION}

In recent years we have learned how to deal with hard exclusive reactions within QCD. In analogy to hard inclusive reactions the process amplitudes factorize in partonic subprocess amplitudes, calculable in perturbation theory, and in GPDs which parameterize soft hadronic matrix elements. In some cases rigorous proofs of factorization exist. For other processes factorization is shown to hold in certain limits, under certain assumptions or is just a hypothesis. This so-called handbag mechanism applies for instance to Compton scattering in kinematical regions where either the virtuality of the incoming photon, $Q^{2}$, is large while the squared invariant moment transfer, $t$, from the initial to thte final proton is small (deeply virtual region) or $-t$ and $-u$ are large but $Q^{2}$ is small (wide-angle region).

The GPDs which are defined by Fourier transforms of bilocal proton matrix elements of quark field operators, describe the emission and reabsorption of partons by the proton. For equal helicities of the emitted and reabsorbed parton the structure of the nucleon is described by four GPDs, termed $H, \widetilde{H}, E$ and $\widetilde{E}$, for each quark flavor and for the gluons. The GPDs are functions of three variables: the momentum transfer, a momentum fraction $x$ and the skewness $\xi$. The latter two variables are related to the individual momentum fractions the emitted and reabsorbed partons carry by

$$
x_{1}=\frac{x+\xi}{1+\xi}, \quad x_{2}=\frac{x-\xi}{1-\xi} .
$$

The GPDs are subject to evolution and, hence, depend on the factorization scale $\mu$. They satisfy the reduction formulas

$$
H^{q}(x, \xi=0, t=0, \mu)=q(x, \mu), \quad \widetilde{H}^{q}(x, \xi=0, t=0, \mu)=\Delta q(x, \mu),
$$

i.e. in the forward limit of zero momentum transfer and zero skewness, $H$ and $\widetilde{H}$ reduce to the usual unpolarized and polarized parton distributions (PDFs), respectively. The forward limits of $E$ and $\widetilde{E}$ are not accessible in deep inelastic electron-proton scattering and are therefore unknown as yet. Another property of the GPDs is the polynomiality which comes about as a consequence of Lorentz covariance

$$
\int_{-1}^{1} d x x^{m-1} H^{q}(x, \xi, t, \mu)=\sum_{i=0}^{[m / 2]} h_{m, i}^{q}(t) \xi^{i},
$$

where $[m / 2]$ denotes the largest integer smaller than or equal to $m / 2$. Eq. (3) holds analogously for the other GPDs and, for $m=1$, implies sum rules for the form factors of the nucleon, e.g.

$$
F_{1}^{q}(t) \equiv h_{1,0}^{q}(t)=\int_{-1}^{1} d x H^{q}(x, \xi, t, \mu)
$$


which represents the contribution of quarks of flavor $q$ to the Dirac form factor of the proton. Reinterpreting as usual a parton carrying a negative momentum fraction $x$ as an antiparton with a positive $x\left(H^{\bar{q}}(x)=-H^{q}(-x)\right)$, one becomes aware that only the difference of the contributions from quarks and antiquarks of given flavor contribute to the sum rules. Introducing the combination

$$
H_{v}^{q}(x, \xi, t, \mu)=H^{q}(x, \xi, t, \mu)-H^{\bar{q}}(x, \xi, t, \mu),
$$

for positive $x$ which, in the forward limit, reduces to the usual valence quark density $q_{v}(x)=q(x)-\bar{q}(x)$, one finds for the Dirac form factor the representation

$$
F_{1}^{p(n)}(t)=e_{u(d)} \int_{0}^{1} d x H_{v}^{u}(x, \xi, t, \mu)+e_{d(u)} \int_{0}^{1} d x H_{v}^{d}(x, \xi, t, \mu) .
$$

Here, $e_{q}$ is the charge of the quark $q$ in units of the positron charge. Possible contributions from other flavors, $s-\bar{s}$ or $c-\bar{c}$, are neglected in the sum rule (6). They are likely small: in the forward limit one has $s(x) \simeq \bar{s}(x)$ [1] and the strange form factors of the nucleon are seemingly small [2]. This simplification of the sum rule (6) does not imply that the nucleon is assumed to consist solely of valence quarks. Sea quarks are there but the virtual photon that probes the quark content of the nucleon, sees only the differences between quark and antiquark distributions which are likely small for strange and charm quarks.

A representation analogue to (6) also holds for the Pauli form factor with $H$ being replaced by $E$. The isovector axial-vector form factor, on the other hand, satisfies the sum rule $\left(\widetilde{H}^{\bar{q}}(x)=\widetilde{H}^{q}(-x)\right)$

$$
F_{A}(t)=\int_{0}^{1} d x\left\{\left[\widetilde{H}_{v}^{u}(x, \xi, t, \mu)-\widetilde{H}_{v}^{d}(x, \xi, t, \mu)\right]+2\left[\widetilde{H}^{\bar{u}}(x, \xi, t, \mu)-\widetilde{H}^{\bar{d}}(x, \xi, t, \mu)\right]\right\},
$$

At least for small $-t$ the magnitude of the second integral in Eq. (7) reflects the size of the flavor non-singlet combination $\Delta \bar{u}(x)-\Delta \bar{d}(x)$ of forward densities. This difference is poorly known, and at present there is no experimental evidence that it might be large [3]. For instance, in the analysis of the polarized PDFs performed by Blümlein and Böttcher Ref. [4] it is zero. Hence, in a perhaps crude approximation the second term in Eq. (7) may be neglected.

\section{EXTRACTING THE ZERO-SKEWNESS GPDS}

Since the GPDs cannot be calculated from QCD with a sufficient degree of accuracy at present we have either to rely on models or to extract them from experiment as it has been done for the PDFs, see for instance Refs. [1, 4]. First attempts to extract the GPDs phenomenologically have been carried through in Refs. [5] and [6]. The main idea is to exploit the sum rules (6) and (7) and to determine the GPDs from the nucleon form factor data, $F_{1}$ and $F_{2}$ for proton and neutron as well as $F_{A}$ (for references to the data see Ref. [5]). Since the sum rules represent only the first moments of the GPDs this task is an ill-posed problem in a strict mathematical sense. Infinitely many moments are needed to deduce the integrand, i.e. the GPDs, unambigously from an integral. However, from phenomenological experience with particle physics one expects the GPDs to be smooth functions and a small number of moments will likely suffice to fix the GPDs. The extreme - and at present the only feasible - point of view is to assume that the lowest moment of a GPD alone is sufficient to fix it [5, 6]. Indeed, using recent results on PDFs [1, 4] and form factor data in combination with physically motivated parameterizations of the GPDs, one can carry through such an analysis. Needless to say that this method while phenomenologically succesful as will be discussed below, does not lead to a unique result. Other parameterizations which may imply different physics, cannot be excluded at the present stage of the art.

The sum rules (6) and (7) hold for all $\xi$ but guessing a plausible parameterization of a function of three variables is nearly hopeless task. In order to simplify matters one may choose the special value $\xi=0$ for which the emitted and reabsorbed partons carry the same momentum fractions. This choice has many advantages. One exclusively works in the so-called DGLAP region where $\xi \leq x$. In this region parton ideas apply, the Fourier transform of the GPDs with respect to the momentum transfer $\Delta\left(\Delta^{2}=-t\right)$ has a density interpretation in the impact parameter plane [7] and, as shown in Refs. [8, 9], wide-angle exclusive processes are controlled by generalized form factors which represent $1 / x$ moments of zero-skewness GPDs. The parameterization that is exploited in Ref. [5] combines the usual PDFs with an exponential $t$ dependence (the arguments $\xi=0$ and $\mu$ are omitted in the following for convenience)

$$
H_{v}^{q}(x, t)=q_{v}(x) \exp \left[t f_{q}(x)\right],
$$



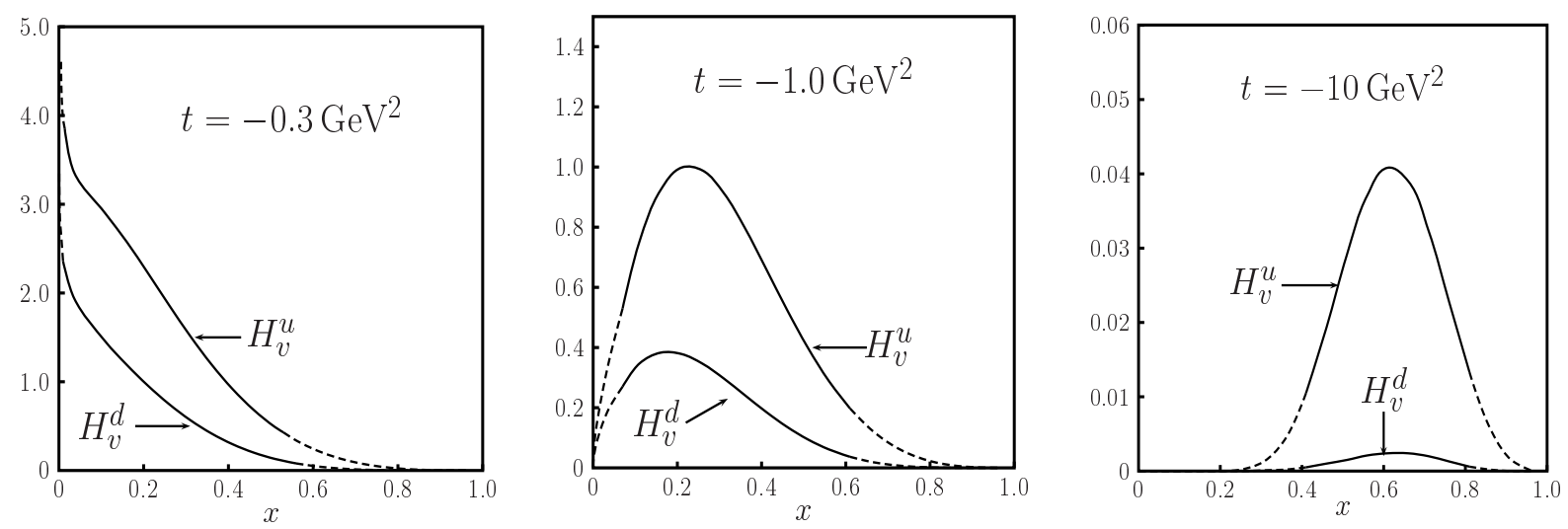

FIGURE 1. Results for $H_{v}^{q}(x, t)$ at the scale $\mu=2 \mathrm{GeV}$ and for $n=2$ obtained in [5]. In each of the regions indicated by dashed lines, $5 \%$ of the total value of the sum rule (6) are accumulated.

where the profile function reads

$$
f_{q}(x)=\left[\alpha^{\prime} \log (1 / x)+B_{q}\right](1-x)^{n+1}+A_{q} x(1-x)^{n} .
$$

This ansatz is motivated by the expected Regge behaviour at low $-t$ and low $x$ [10] (where $\alpha^{\prime}$ is the Regge slope for which the value $0.9 \mathrm{GeV}^{2}$ is imposed at low factorization scales). At large $-t$ and large $x$, on the other hand, one expects a behaviour like $f_{q} \sim 1-x$ from overlaps of light-cone wavefunctions $[8,11]$. The ansatz (8), (9) interpolates between the two limits smoothly ${ }^{1}$ and allows for a stronger suppression of $f_{q}$ in the limit $x \rightarrow 1$. It matches the following criteria for a good parameterization: simplicity, consistency with theoretical and phenomenological constraints, stability with respect to variations of PDFs and stability under evolution (scale dependence of the GPDs can be absorbed into parameters).

Using the CTEQ PDFs [1], one obtains a reasonable fit of the ansatz (8), (9) to the data on the Dirac form factor which range from $-t=0$ to $\simeq 30 \mathrm{GeV}^{2}$, with the parameters

$$
\begin{aligned}
& B_{u}=B_{d}=(0.59 \pm 0.03) \mathrm{GeV}^{-2}, \\
& A_{u}=(1.22 \pm 0.020) \mathrm{GeV}^{-2}, \quad A_{d}=(2.59 \pm 0.29) \mathrm{GeV}^{-2},
\end{aligned}
$$

quoted for the case $n=2$ and at a scale of $\mu=2 \mathrm{GeV}$. In Fig. 1 the results for $H_{v}^{q}$ are shown at three values of $t$. While at small $-t$ the behaviour of the GPD still reflects that of the parton densities it exhibits a pronounced maximum at larger values of $-t$. The maximum becomes more pronounced with increasing $-t$ and its position moves towards $x=1$. In other words only a limited range of large $x$ contributes to the form factor substantially. The quality of the fit is very similar in both the cases, $n=1$ and 2; the results for the GPDs and related quantities agree well with each other. Substantial differences between the two results only occur at very low and very large values of $x$, i.e. in the regions which are nearly insensitive to the present form factor data. It is the physical interpretation of the results which favours the fit with $n=2$. Indeed the average distance between the struck quark and the cluster of spectators becomes unphysical large for $x \rightarrow 1$ in the case $n=1$; it grows like $\sim 1 /(1-x)$ while, for $n=2$, it tends towards a constant value of about $0.5 \mathrm{fm} \mathrm{[5].}$

The analysis of the axial and Pauli form factors, with parameterizations analogue to Eqs. (8), (9), provides the GPDs $\widetilde{H}$ and $E$. They behave similar to $H$, see Fig. 2. Noteworthy differences are the opposite signs of $\widetilde{H}_{v}^{u}\left(E_{v}^{u}\right)$ and $\widetilde{H}_{v}^{d}\left(E_{v}^{d}\right)$ and the relative magnitude of $E_{v}^{u}$ and $E_{v}^{d}$. At intermediate values of $-t$ the GPD $-E_{v}^{d}$ develops a maximum which is more pronounced and located at significantly smaller $x$ than in $E_{v}^{u}$. At larger values of $-t\left|E_{v}^{d}\right|$ becomes gradually smaller than $E_{v}^{u}$. For $H_{v}^{q}$ and $\widetilde{H}_{v}^{q}$, on the other hand, the $d$-quark contributions are substantially smaller in magnitude than the $u$-quark ones, see Fig. 1. Since there is no data available for the pseudoscalar form factor of the nucleon the GPD $\widetilde{E}$ cannot be determined this way.

\footnotetext{
1 The parameter $B_{q}$ is not needed if $\alpha^{\prime}$ is freed. A fit to the data of about the same quality and with practically the same results for the GPDs is obtained for $\alpha^{\prime} \simeq 1.4$.
} 

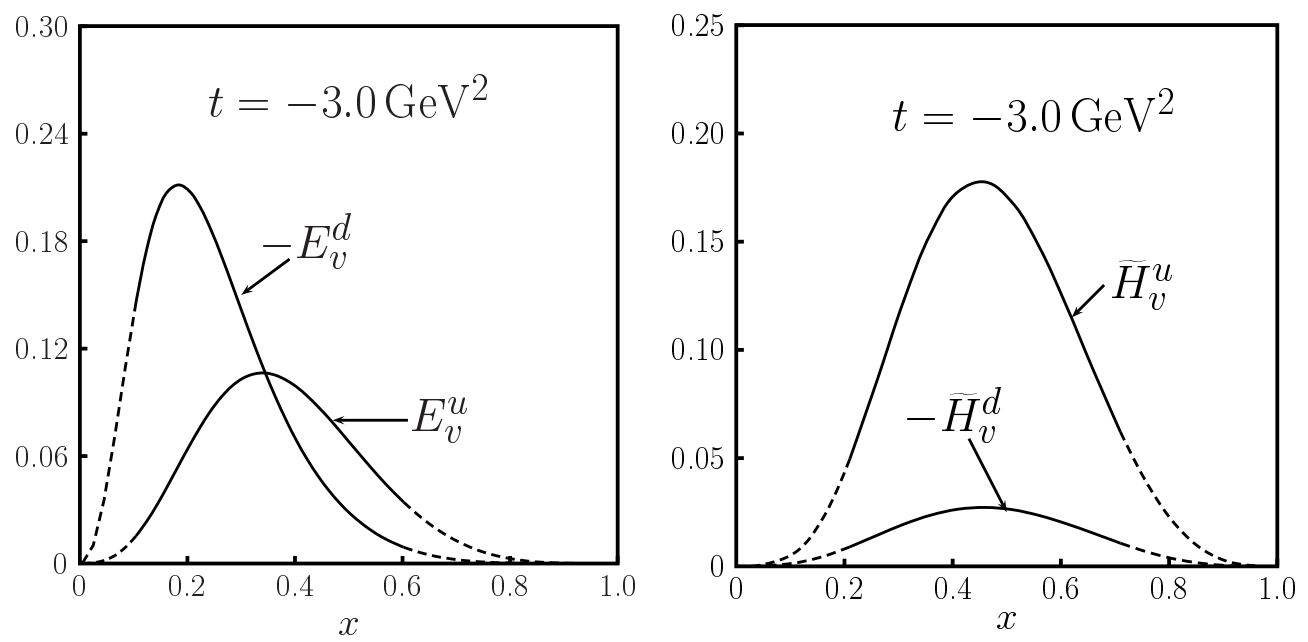

FIGURE 2. The GPDs $E_{v}^{u(d)}$ (left) and $\widetilde{H}_{v}^{u(d)}$ (right) at the scale $\mu=2 \mathrm{GeV}$.
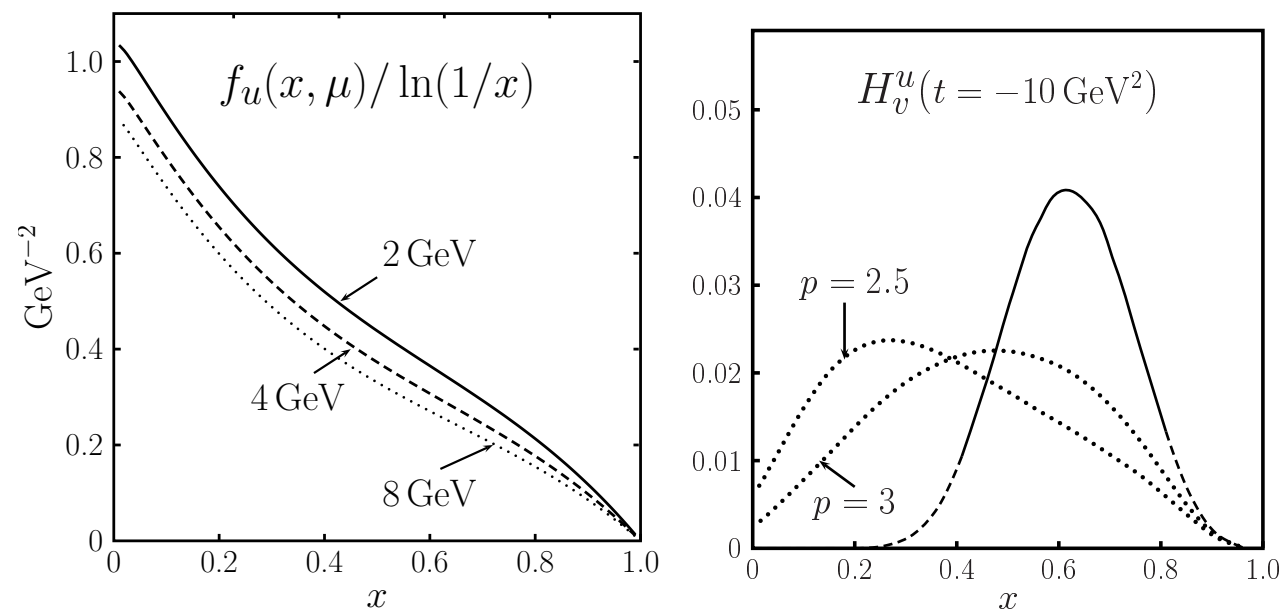

FIGURE 3. The scale dependence of the profile function for $u$ quarks (left) and the $H_{v}^{u}$ at $t=-10 \mathrm{GeV}^{2}$ obtained with the parameterization (11) for the powers $p=2.5$ and 3 (right). These results are compared to the GPD obtained with the exponential profile function (8), (9).

The scale dependence of the profile function for $u$ quarks is shown in Fig. 3. Combined with that of the PDF it provides the scale dependence of $H^{u}$. Analogue results are found for $d$ quarks and for the other GPDs. The profile functions decrease with increasing $\mu$, i.e., the nucleon becomes more compact in the impact parameter plane at larger scales.

Up to now only the sum rules (6), (7) have been utilized in the GPD analysis. As mentioned above, in this situation parameterizations of the GPDs are required with the consequence of non-unique results. An alternative ansatz [5] is for instance

$$
H_{v}^{q}(x, t)=q_{v}(x)\left[1-t f_{q}(x) / p\right]^{-p} .
$$

Although reasonable fits to the form factors are obtained with (11) for $p \gtrsim 2.5$ it is physically less appealing than the parameterization (8): the combination of Regge behaviour at small $x$ and $t$ with the dynamics of the Feynman mechanism is lost. The resulting GPDs have a broader shape and $H(x=0, t)$ remains finite, see Fig. 3. Thus, small $x$ also contribute to the high- $t$ form factors for this parameterization. The parameterization (11) is not stable under DGLAP evolution. 

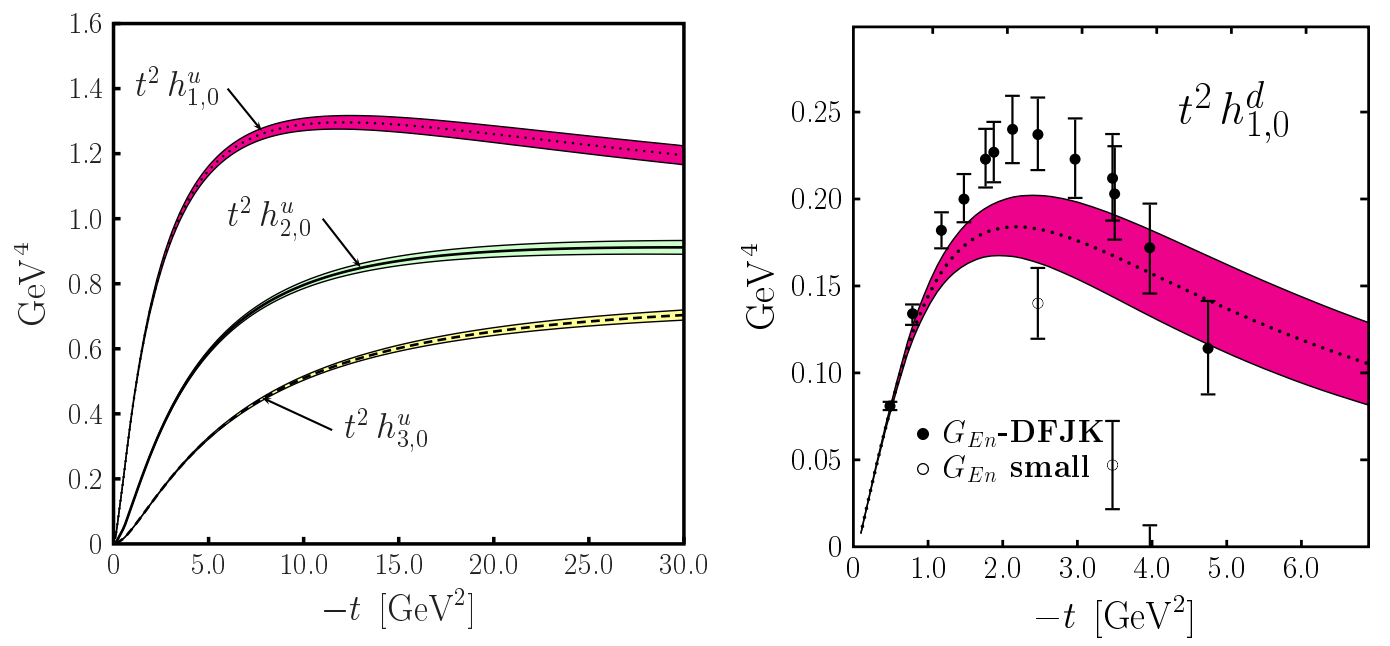

FIGURE 4. The first three moments of $H_{v}^{u}$ (left) and the lowest moment of $H_{v}^{d}$ (right) scaled by $t^{2}$. The shaded bands represent the parametric uncertainties [5]. Data are taken from Ref. [13] and from the references quoted in [5]. Results for two different extrapolations of $G_{E}^{n}$ are shown.

\section{APPLICATIONS}

Having the GPDs at disposal one can evaluate various moments, some of them are displayed in Fig. 4. An interesting property of the moments is that the $u$ and $d$ quark contributions decrease with different rates at large $-t$. Since in this region the dominant contribution to the form factors comes from a narrow region of large $x$ (see Fig. 1) one can use the large $x$ approximations (given for the favored case $n=2$ )

$$
q_{v} \sim(1-x)^{\beta_{q}}, \quad f_{q} \sim A_{q}(1-x)^{2},
$$

and evaluate the sum rule (6) in the saddle point approximation. This leads to

$$
h_{1,0}^{q} \sim|t|^{-\left(1+\beta_{q}\right) / 2}, \quad 1-x_{s}=\left(\frac{2}{\beta_{q}} A_{q}|t|\right)^{-1 / 2},
$$

where $x_{s}$ is the position of the saddle point. The latter lies within the region where the GPD is large and using the the CTEQ values for $\beta_{q}\left(\beta_{u} \simeq 3.4\right.$ and $\beta_{d} \simeq 5$ [1]), one obtains a fall off slightly faster than $t^{-2}$ for the form factor $h_{1,0}^{u}$ while the $d$-quark form factor falls as $t^{-3}$. Strengthened by the charge factor the $u$-quark contribution consequently dominates the proton's Dirac form factor ${ }^{2}$ for $-t$ larger than about $5 \mathrm{GeV}^{2}$, the $d$-quark contribution amounts to less than $10 \%$. The power behaviour (13) bears resemblance to the Drell-Yan relation [12]. In fact the common underlying dynamics is the Feynman mechanism which applies in the soft region where $1-x \sim \Lambda / \sqrt{-t}$ and the virtualities of the active partons are $\sim \Lambda \sqrt{-t}$ ( $\Lambda$ is a typical hadronic scale of order $1 \mathrm{GeV}^{2}$ ), cf. Eq. (13). The Drell-Yan relation is, however, an asymptotic result $(x \rightarrow 1, t \rightarrow-\infty)$ which bases on the assumption of valence Fock state dominance, i.e. on the absence of sea quarks. The different powers for $u$ and $d$ quarks signal that the asymptotic region where the dimensional counting rules apply, has not yet been reached.

One may object to the different powers that they are merely a consequence of the chosen parameterization (8), (9). However, this is likely not the case. The moments $h_{1,0}^{q}$ can directly be extracted from the form factor data. The experimental results for the $d$-quark moment are shown in Fig. 4. The sharp fall off with increasing $-t$ is clearly visible. The small deviations between data and the moment obtained in [5] are caused by the very precise but still preliminary CLAS data [13] on $G_{M}^{n}$. These data have been utilized in the extraction of the moments $h_{1,0}^{q}$ but not in the analysis in Ref. [5]. Worst measured of the four form factors is the electric one of the neutron. Above $1.5 \mathrm{GeV}^{2}$ no data

2 This implies the ratio $F_{1}^{n} / F_{1}^{p}=e_{d} / e_{u}$ at large $-t$. 

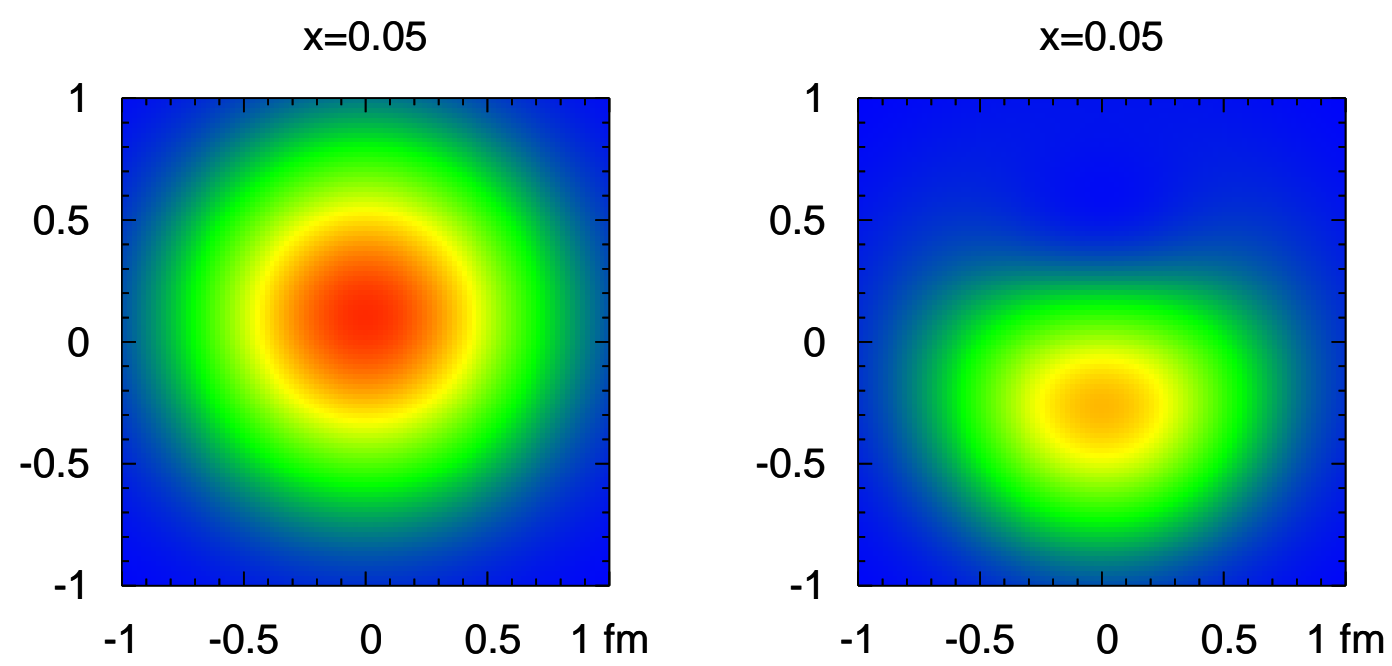

FIGURE 5. The $u$ (left) and $d$ (right) quark densities in the impact parameter plane at $x=0.005$. The proton is polarixed in $X$ direction, see text.

is available as yet and two different extrapolations to larger $-t$ are used in the extraction of the moments $h_{1,0}^{q}$. Thus, except $G_{E}^{n}$ differs from expectation strongly, the rapid decrease of $h_{1,0}^{d}$ seen in Fig. 4, seems to be an experimental fact. Future JLab data on $G_{E}^{n}$ will settle this question.

A combination of the second moments of $H$ and $E$ at $t=0$ is Ji's sum rule [14] which allows for an evaluation of the valence quark contribution to the orbital angular momentum the quarks inside the proton carry

$$
\left\langle L_{v}^{q}\right\rangle=\frac{1}{2} \int_{0}^{1} d x\left[x E_{v}^{q}(x, t=0)+x q_{v}(x)-\Delta q_{v}(x)\right] .
$$

The analysis of the three GPDs leads to

$$
\left\langle L_{v}^{u}\right\rangle=-(0.24-0.27), \quad\left\langle L_{v}^{d}\right\rangle=0.15-0.19,
$$

for the valence quark contributions to the orbital angular momentum at a scale of $\mu=2 \mathrm{GeV}$. The signs of these predictions are in accord with recent results from lattice QCD $[15,16]$ but the absolute values are somewhat larger. Given the uncertainties in all these analyses, Ref. [5] and the lattice calculations, one may ascertain fair agreement. There is also remarkable agreement of the $t$ dependencies of the moments obtained in Ref. [5] and in lattice QCD studies.

While the parton distributions only provide information on the longitudinal distribution of quarks inside the nucleon, GPDs also give access to the transverse position distributions of partons within the proton. Thus, the Fourier transform of $H$ for instance

$$
q_{v}(x, \mathbf{b})=\int \frac{d^{2} \Delta}{(2 \pi)^{2}} e^{-i \mathbf{b} \cdot \Delta} H_{v}^{q}\left(x, t=-\Delta^{2}\right),
$$

gives the probability of finding a valence quark with longitudinal momentum fraction $x$ and impact parameter $\mathbf{b}$ as seen in a frame in which the proton moves rapidly in the $z$ direction. Together with the analogue Fourier transform of $E_{v}^{q}(x, t)$ one can form the combination ( $m_{p}$ being the mass of the proton)

$$
q_{v}^{X}(x, \mathbf{b})=q_{v}(x, \mathbf{b})-\frac{b^{Y}}{m_{p}} \frac{\partial}{\partial \mathbf{b}^{2}} e_{v}^{q}(x, \mathbf{b}),
$$

which gives the probability to find an unpolarized valence quark with momentum fraction $x$ and impact parameter $\mathbf{b}=\left(b^{X}, b^{Y}\right)$ in a proton that moves rapidly along the $Z$ direction and is polarized in $X$ direction [7]. As shown in Ref. [5], for small $x$ one observes a very broad distribution while at large $x$ it becomes more focussed on the center of momentum defined by $\sum_{i} x_{i} \mathbf{b}_{i}=0\left(\sum_{i} x_{i}=1\right)$. In a proton that is polarized in the $X$ direction the symmetry around the $Z$ axis is lost and the center of the density is shifted in the $Y$ direction away from the center of momentum, downward 

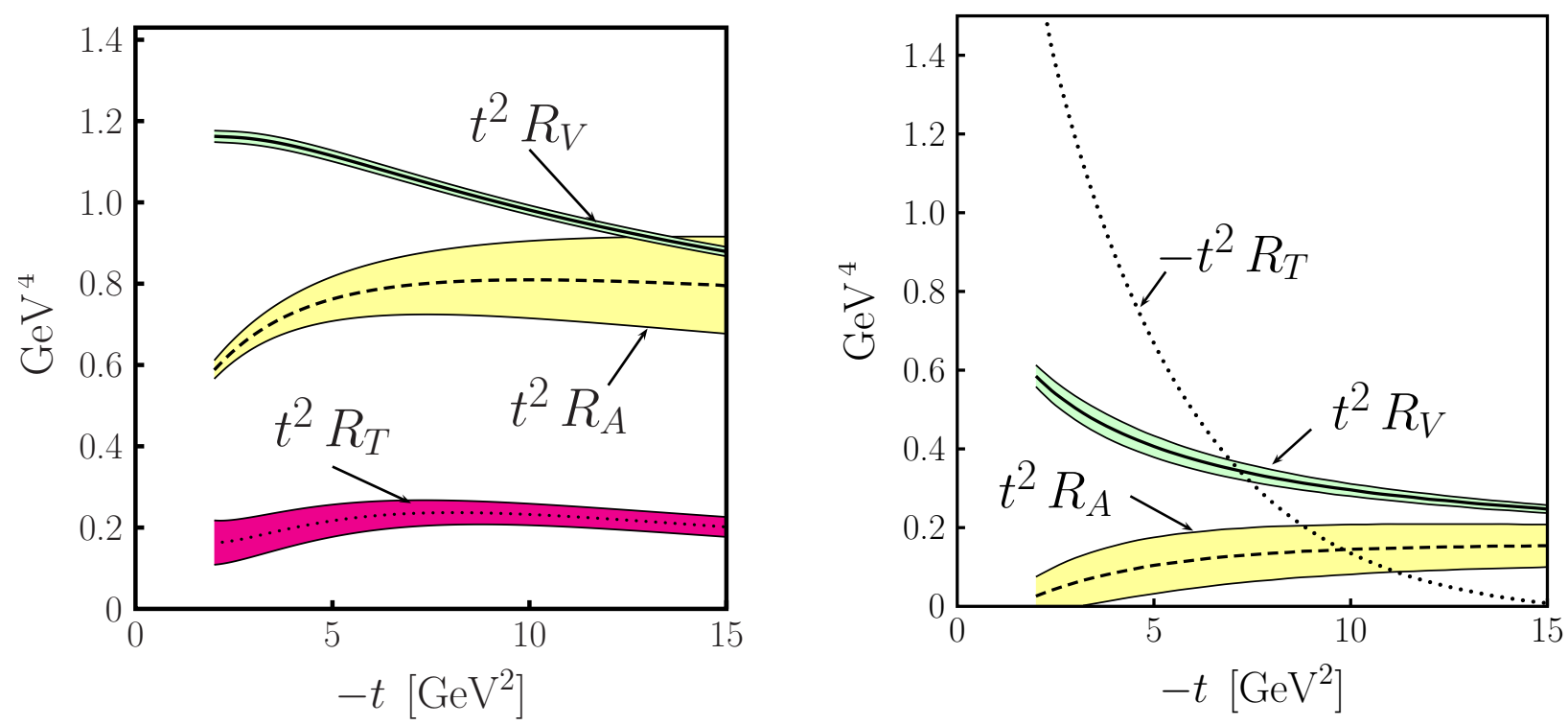

FIGURE 6. The Compton form factors for protons (left) and neutrons (right), scaled by $t^{2}$, evaluated from the GPDs determined in Ref. [5]. The bands represent the parametric uncertainties of the form factors. For the tensor form factor of the neutron the error band is not shown for clearness.

for $d$ quarks and upward for $u$ ones. Thus, a polarization of the proton induces a flavor segregation in the direction orthogonal to the direction of the polarization and the proton momentum, see Fig. 5. This effect may be responsible for the Sivers function [17], $f_{1 T}^{\perp}$. One may argue [18] that the average Sivers function for quarks of flavor $q$ is related to the contribution of these quarks to the anomalous magnetic moment by

$$
f_{1 T}^{\perp q} \sim-\kappa_{q}
$$

where

$$
\kappa_{q}=\int_{0}^{1} d \bar{x} E_{v}^{q}(\bar{x}, 0,0)
$$

The anomalous moments of the quarks are linear combination of those of proton and neutron

$$
\kappa_{u}=2 \kappa_{p}+\kappa_{n} \simeq 1.67, \quad \kappa_{d}=\kappa_{p}+2 \kappa_{n} \simeq-2.03 .
$$

This explains the different signs of the Sivers functions fir $u$ and $d$ quarks and is also the origin of the opposite signs of $E_{v}^{u}$ and $E_{v}^{d}$ (see Fig. 2). The opposite signs of the Sivers functions is in agreement with a recent HERMES measurement [19].

\section{WIDE-ANGLE COMPTON SCATTERING}

As discussed in previous sections the analysis of the GPDs gives insight in the transverse distribution of quarks inside the proton. However, there is more in it. The universality property of the GPDs, i.e. their process independence, allows to predict other hard exclusive reactions once the GPDs have been determined in the analysis of a given process. This way QCD acquires a predictive power for hard exclusive processes provided factorization holds.

Thus, with the $\xi=0$ GPDs at hand one can predict hard wide-angle exclusive reactions like Compton scattering or meson photo- and electroproduction. For these reactions it is advantagous to work in a so-called symmetrical frame where the skewness is zero. It has been argued [8,9] that, for large Mandelstam variables $\left(s,-t,-u \gg \Lambda^{2}\right)$, the amplitudes for these processes factorize in a hard partonic subprocess, e.g. Compton scattering off quarks and in form factors representing 1/ $x$-moments of zero-skewness GPDS (see Fig. 5). For Compton scattering off protons these form factors read 


$$
\begin{aligned}
& R_{V}(t) \simeq \sum_{q=u, d} e_{q}^{2} \int_{0}^{1} \frac{d x}{x} H_{v}^{q}(x, t), \\
& R_{A}(t) \simeq \sum_{q=u, d} e_{q}^{2} \int_{0}^{1} \frac{d x}{x} \widetilde{H}_{v}^{q}(x, t), \\
& R_{T}(t) \simeq \sum_{q=u, d} e_{q}^{2} \int_{0}^{1} \frac{d x}{x} E_{v}^{q}(x, t) .
\end{aligned}
$$

In these expressions contributions from sea quarks have been neglected ${ }^{3}$. An analogue pseudoscalar form factor related to the GPD $\widetilde{E}$, decouples in the symmetric frame. Numerical results for the Compton form factors off protons and neutrons are shown in Fig. 6. The latter are obtained from Eq. (21) with the help of isospin invariance: $H^{d(u)}$ for the neutron equals $H^{u(d)}$ for the proton (see Eq. (6)). The axial-vector and the tensor form factors behave differently for protons and neutrons. This is a consequence of the opposite signs for the $u$ and $d$ quark contributions (see Fig. 2) in combination with the different charge weigths. For smaller values of $-t$ the form factor $R_{A}$ cancels almost completely in the case of the neutron. Since, at intermediate $-t,\left|E_{v}^{d}\right|$ exhibits a maximum that is more pronounced and located at smaller values of $x$ than that of $E_{v}^{u}$ (see Fig. 2), its $1 / x$ moment is larger than that of the latter one. In combination with the quark charges this leads to due to a strong cancellation in the case of the proton and, hence, to a very small form factor $R_{T}$. For the neutron, on the other hand, the properties of $E$ entaila a form factor $R_{T}$ which is negative and large in absolute value at intermediate $-t$.

The handbag contribution leads to the following leading-order result for the Compton cross section $[8,20]$

$$
\begin{aligned}
\frac{d \sigma}{d t}= & \frac{d \hat{\sigma}}{d t}\left\{\frac{1}{2}\left[R_{V}^{2}(t)+\frac{-t}{4 m_{p}^{2}} R_{T}^{2}(t)+R_{A}^{2}(t)\right]\right. \\
& \left.-\frac{u s}{s^{2}+u^{2}}\left[R_{V}^{2}(t)+\frac{-t}{4 m_{p}^{2}} R_{T}^{2}(t)-R_{A}^{2}(t)\right]\right\},
\end{aligned}
$$

where $d \hat{\sigma} / d t$ is the Klein-Nishina cross section for Compton scattering off massless, point-like spin-1/2 particles of charge unity. Next-to-leading order QCD corrections to the subprocess have been calculated in Ref. [20]. They are not displayed in (22) but taken into account in the numerical results presented below.

Inserting the Compton form factors (21) into Eqs. (22), one can predict the Compton cross section in the wide-angle region. The results for sample values of $s$ are shown in Fig. 7 and compared to recent measurements from JLab [21]. The inner bands of the predictions for $d \sigma / d t$ reflect the parametric errors of the form factors, essentially that of the vector form factor which dominates the cross section. The outer bands indicate the size of target mass corrections, see Ref. [22]. In order to comply with the kinematical requirements for handbag factorization, at least in a minimal fashion, predictions are only shown for $-t$ and $-u$ larger than about $2.4 \mathrm{GeV}^{2}$. Fair agreement between theory and experiment is to be seen. Predictions for Compton scattering off neutrons are also shown in Fig. 7. In the case of the neutron the uncertainties of the form factors dominate. In the forward hemisphere the cross section is large, comparable with the proton cross section because the tensor form factors is so large, see Fig. 6. In the backward hemisphere, with increasing $-t$ the neutron cross section becomes small, tending towards the suppression factor $\left(e_{d} / e_{u}\right)^{4}=1 / 16$ which limit is obvious in the light of the discussion below Eq. (13). A measurement of $\gamma n \rightarrow \gamma n$ will provide a severe test of our present understanding of the GPD $E$.

With the Compton form factors at hand also other Compton observables can be calculated in a parameter-free way. Thus, for instance, the helicity correlation, $A_{L L}$, between the initial state photon and nucleon or, equivalently, the helicity transfer, $K_{L L}$, from the incoming photon to the outgoing nucleon. In the handbag approach one obtains [20]

$$
A_{L L}=K_{L L}=\frac{s^{2}-u^{2}}{s^{2}+u^{2}} \frac{R_{A}(t)}{R_{V}(t)} \frac{1+\beta \sqrt{-t} /\left(2 m_{p}\right) R_{T} / R_{V}}{1-t /\left(4 m_{p}^{2}\right) R_{T}^{2} / R_{V}^{2}}\left[1+\frac{R_{A}^{2}-R_{V}^{2}\left(1-t /\left(4 m_{p}^{2}\right) R_{T}^{2} / R_{V}^{2}\right)}{2 R_{V}^{2}\left(1-t /\left(4 m_{p}^{2}\right) R_{T}^{2} / R_{V}^{2}\right)} \frac{t^{2}}{s^{2}+u^{2}}\right]^{-1}
$$

\footnotetext{
${ }^{3}$ An estimate of the sea quark contributions may be obtained by using the ansatz (8) with the same profile function for the sea quarks as for the valence ones but replacing the valence quark density with the CTEQ [1] antiquark ones. The so estimated contributions are indeed small.
} 

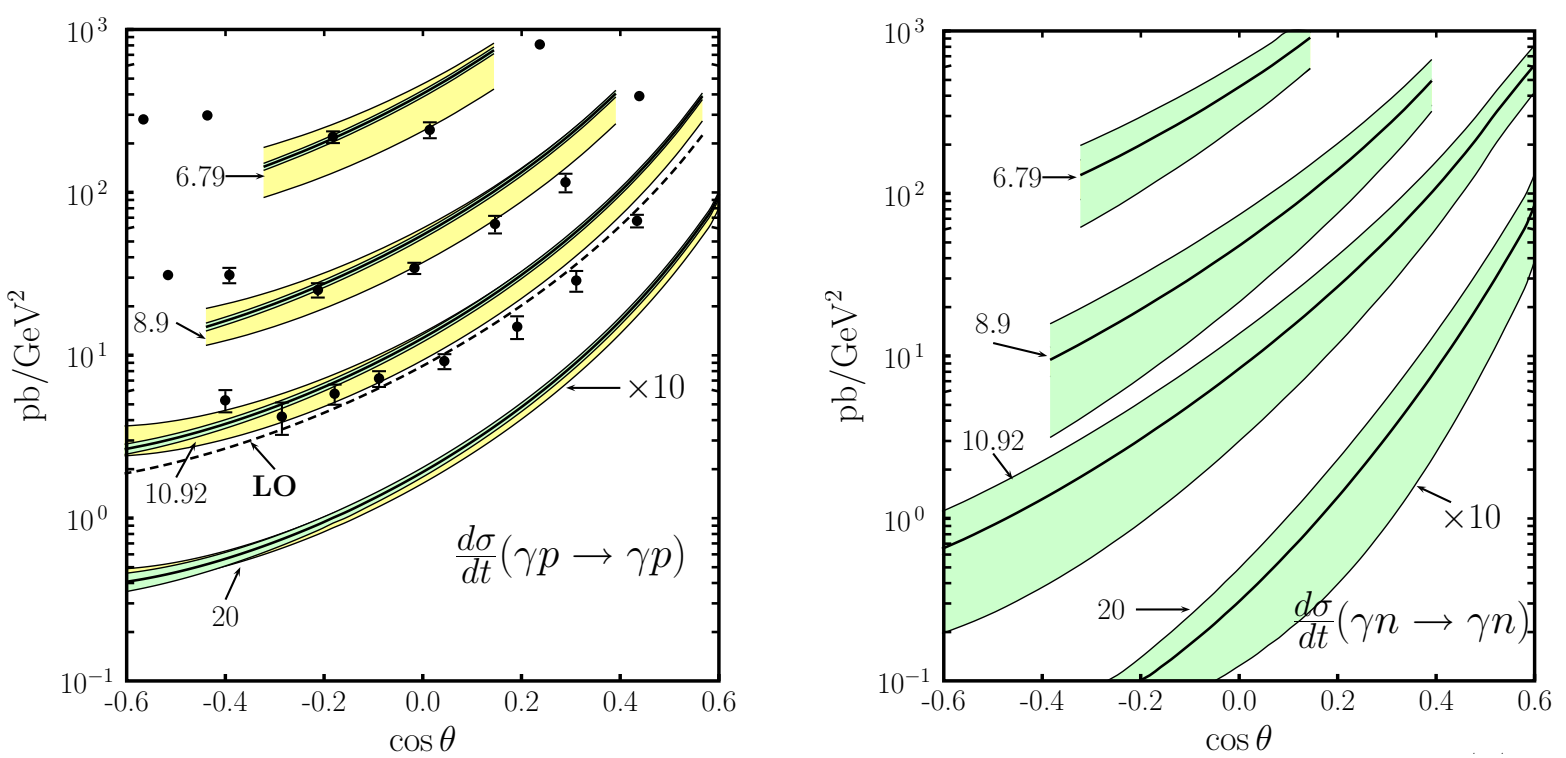

FIGURE 7. The cross sections for Compton scattering off protons (left) and neutrons (right) versus the c.m.s. scattering angle $\theta$ at $s=6.9,8.9,11$ and $20 \mathrm{GeV}^{2}$. The error bands are explained in the text. Data are taken from Ref. [21].

where the factor in front of the form factors is the corresponding observable for $\gamma q \rightarrow \gamma q\left(\hat{A}_{L L}\right)$. The kinematical factor $\beta$ reads $2 m_{p} / \sqrt{s} \sqrt{-t} /(\sqrt{s}+\sqrt{-u})$. For Compton scattering off the proton Eq. (23) can be approximated by

$$
A_{L L}=K_{L L} \simeq \frac{s^{2}-u^{2}}{s^{2}+u^{2}} \frac{R_{A}(t)}{R_{V}(t)}
$$

The latter result is a robust prediction of the handbag mechanism, the magnitude of the subprocess helicity correlation, $\hat{A}_{L L}$, is only diluted somewhat by the ratio of the form factors $R_{A}$ and $R_{V}$. It is to be stressed that $A_{L L}$ and $K_{L L}$ are identically in the handbag approach because the quarks are assumed to be massless and consequently there is no quark helicity flip. For an alternative approach, see Ref. [23]. For Compton scattering off neutrons $A_{L L}$ is very small at JLab energies since $R_{T}$, neglected in Eq. (24), is large in this case.

The JLab Hall A collaboration [24] has presented a first measurement of $K_{L L}$ at $s=6.9 \mathrm{GeV}^{2}$ and $t=-4 \mathrm{GeV}^{2}$. The kinematical requirement of the handbag mechanism, $s,-t,-u \gg m^{2}$, is not satisfied for this measurement since $-u$ is only $1.13 \mathrm{GeV}^{2}$. One therefore has to be very cautious when comparing this experimental result with the handbag predictions, there might be large dynamical and kinematical corrections. Nevertheless the agreement of this data point with the prediction from the handbag approach is good which may be considered as a non-trivial and promising fact. Polarization data at higher energies are desired as well as a measurement of the angle dependence.

The handbag approach also applies to wide-angle photo- and electroproduction of pseudoscalar and vector mesons [25]. The amplitudes again factorize into a parton-level subprocess, $\gamma q \rightarrow M q$ now, and form factors which represent $1 / x$-moments of GPDs. Their flavor decomposition differs from those appearing in Compton scattering. It now reflects the valence quark content of the produced meson. Since the GPDs and, hence, the form factors for a given flavor, $R_{i}^{q}, i=V, A, T$ are process independent they are known from the analysis of Ref. [5] for $u$ and $d$ quarks. Therefore, the form factors occuring in photo-and electroproduction of pions and $\rho$ mesons within the handbag approach can also be evaluated from the GPDs given in Ref. [5].

\section{SUMMARY AND OUTLOOK}

Results from a first analysis of the GPDs at zero skewness have been reviewed. The analysis, performed in analogy to those of the usual parton distributions, bases on a physically motivated parameterization of the GPDs with a few free parameters fitted to the available nucleon form factor data. The analysis provides results on the valence-quark GPDs $H, \widetilde{H}$ and $E$. The distribution of the quarks in the impact parameter plane transverse to the direction of the nucleon's 


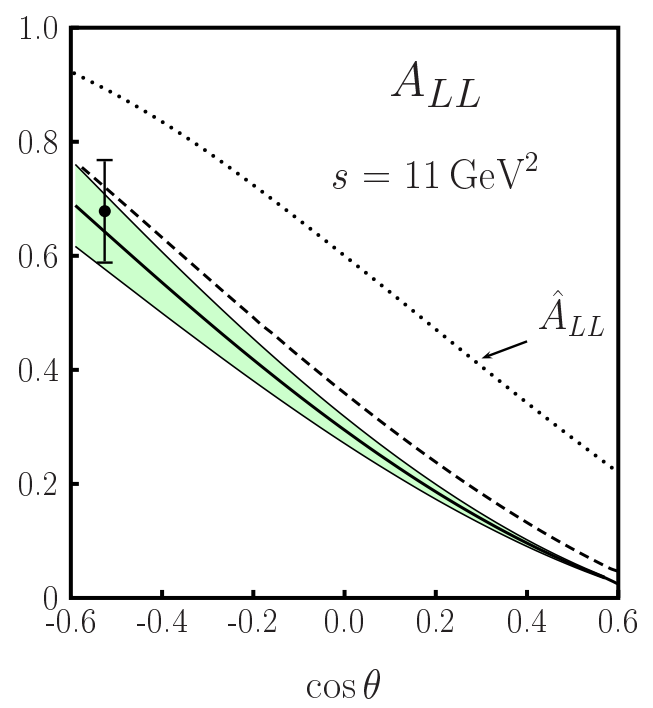

FIGURE 8. Handbag prediction for the helicity correlation $A_{L L}=K_{L L}$ in Compton scattering off protons at $s=11 \mathrm{GeV}^{2}$ (right). Data are taken from Ref. [24]. The green band represents the uncertainties of the handbag prediction due to the errors of the Compton form factors.

momentum can be evaluated from them. Polarizing the nucleon induces a flavor segregation in the direction orthogonal to the those of the nucleon's momentum and of its polarization. The average orbital angular momentum of the valence quarks can be estimated from the obtained GPDs, too.

The zero-skewness GPDs form the soft physics input to hard wide-angle exclusive reactions. For Compton scattering, for instance, the soft physics is encoded in specific form factors which represent $1 / x$ moments of zero-skewness GPDs. Using the GPD determined in Ref. [5], one can evaluate the form factors and predict the wide-angle Compton cross section. The results are found to be in good agreement with experiment.

An analysis as that one performed in Ref. [5] can only be considered as a first attempt towards a determination of the GPDs. It needs improvements in various aspects. High quality data on the form factors at larger $t$ are required in order to stabilize the parameters. JLab will provide such data in the near future. As already mentioned CLAS [13] will come up with data on $G_{M}^{n}$ up to about $5 \mathrm{GeV}^{2}$. $G_{E}^{n}$ will be measured up to about $4 \mathrm{GeV}^{2}$ next year and $G_{E}^{p}$ up to $9 \mathrm{GeV}^{2}$ in 2007. The upgraded Jlab will allow measurements of the nucleon form factors up to about $13 \mathrm{GeV}^{2}$. Data on the axial form factor are needed in order to improve our knowledge of $\widetilde{H}$.

Up to now only the sum rules (6), (7) have been utilized in the GPD analysis. This, as discussed above, does not lead to a unique solution for the GPDs. Higher moments are needed in order to lessen the dependence on the chosen parameterization for the GPDs. Such moments can be provided by lattice gauge theories. The present lattice results $[15,16]$ are however calculated in scenarios where the pion is heavy (typically $600-800 \mathrm{MeV}$ ) and the extrapolation to the chiral limit is not performed. Obviously such results are inappropriate for a GPD analysis. In a few years however the quality of the moments from lattice gauge theories may suffice. It is also tempting to use the data on wide-angle Compton scattering [21] which provide information on 1/x moments of the GPDs. Even at the highest measured energy, $s=11 \mathrm{GeV}^{2}$, the Compton cross section may still be contaminated by power corrections rendering its use in a GPD analysis dubious. For data at, say, $s=20 \mathrm{GeV}^{2}$ the situation may be different.

Up to now only the zero-skewness GPDs have been determined. Although the sum rules (6), (7) holds for all values of the skewness it seems hopeless task to extract the GPDs as funtions of three variables from them. Additional information is demanded in the case on $\xi \neq 0$ and will be provided by deeply virtual exclusive scattering (DVES).

\section{ACKNOWLEDGEMENTS}

It is a pleasure to thank Aaron Bernstein and Costas Papanicolas for organising this interesting meeting and for the hospitality extended to the author in Athens. 


\section{REFERENCES}

1. J. Pumplin et al [CTEQ collaboration], JHEP 0207, 012 (2002).

2. F. E. Maas et al., Phys. Rev. Lett. 94, 152001 (2005); K. A. Aniol et al. [HAPPEX Collaboration], nucl-ex/0506011; D. S. Armstrong et al. [G0 Collaboration], Phys. Rev. Lett. 95, 092001 (2005).

3. A. Airapetian et al. [HERMES Collaboration], Phys. Rev. Lett. 92, 012005 (2004).

4. J. Blümlein and H. Böttcher, Nucl. Phys. B636, 225 (2002).

5. M. Diehl, T. Feldmann, R. Jakob and P. Kroll, Eur. Phys. J. C 39, 1 (2005).

6. M. Guidal, M. V. Polyakov, A. V. Radyushkin and M. Vanderhaeghen, Phys. Rev. D 72, 054013 (2005).

7. M. Burkardt, Int. J. Mod. Phys. A 18, 173 (2003).

8. M. Diehl, T. Feldmann, R. Jakob and P. Kroll, Eur. Phys. J. C 8, 409 (1999).

9. A. V. Radyushkin, Phys. Rev. D 58, 114008 (1998).

10. H.D.I. Arbarbanel, M.L. Goldberger and S.B. Treiman, Phys. Rev. Lett. 22, 500 (1969); P.V. Landshoff, J.C. Polkinghorne and R.D. Short, Nucl. Phys. B 28, 225 (1971).

11. M. Diehl, T. Feldmann, R. Jakob, and P. Kroll, Nucl. Phys. B 596, 33 (2001), Erratum-ibid. 605, 647 (2001).

12. S. D. Drell and T. M. Yan, Phys. Rev. Lett. 24, 181 (1970).

13. W. K. Brooks and J. D. Lachniet, Nucl. Phys. A 755, 261 (2005).

14. X.-D. Ji, Phys. Rev. Lett. 78, 610 (1997).

15. P. Hägler, J. W. Negele, D. B. Renner, W. Schroers, T. Lippert and K. Schilling [LHPC Collaboration], Eur. Phys. J. A 24S1, 29 (2005).

16. M. Gockeler, R. Horsley, D. Pleiter, P. E. L. Rakow, A. Schafer, G. Schierholz and W. Schroers [QCDSF Collaboration], Phys. Rev. Lett. 92, 042002 (2004).

17. D.W. Sivers, Phys. Rev. D 43, 261 (1991).

18. M. Burkardt, Phys. Rev. D 72, 094020 (2005).

19. HERMES collaboration, Phys. Rev. Lett. 94, 012002 (2005).

20. H. W. Huang, P. Kroll and T. Morii, Eur. Phys. J. C 23, 301 (2002)[Erratum-ibid. C 31, 279 (2003)].

21. A. Danagulian, A. M. Nathan, M. Roedelbronn, D. J. Hamilton, C. E. Hyde-Wright, V. H. Mamian and B. Wojtsekhowski, Nucl. Phys. A 755, 281 (2005); V. H. Mamian et al., proceedings of the Armenyan Academy of Science, Physics, 40, 325 (2005).

22. M. Diehl, T. Feldmann, H. W. Huang and P. Kroll, Phys. Rev. D 67, 037502 (2003).

23. G. A. Miller, Phys. Rev. C 69, 052201 (2004).

24. D. J. Hamilton et al. [Jefferson Lab Hall A Collaboration], Phys. Rev. Lett. 94, 242001 (2005).

25. H. W. Huang and P. Kroll, Eur. Phys. J. C 17, 423 (2000);H. W. Huang, R. Jakob, P. Kroll and K. Passek-Kumericki, Eur. Phys. J. C 33, 91 (2004). 